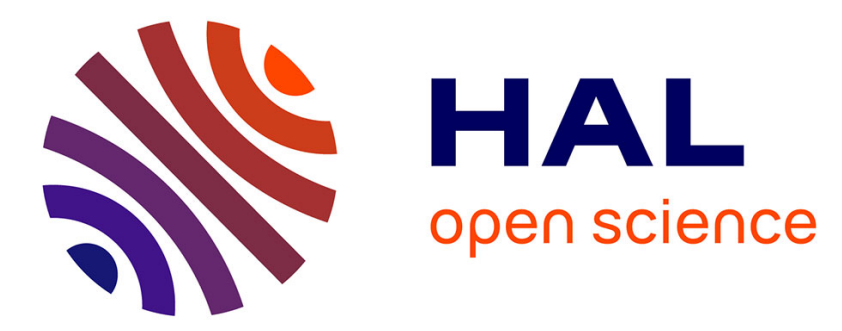

\title{
Robust object characterization from lensless microscopy videos
}

Olivier Flasseur, Loic Denis, Corinne Fournier, Thiébaut Eric

\section{To cite this version:}

Olivier Flasseur, Loic Denis, Corinne Fournier, Thiébaut Eric. Robust object characterization from lensless microscopy videos. 25th European Signal Processing Conference, Aug 2017, Kos Island, Greece. ujm-01626086

\section{HAL Id: ujm-01626086 \\ https://hal-ujm.archives-ouvertes.fr/ujm-01626086}

Submitted on 30 Oct 2017

HAL is a multi-disciplinary open access archive for the deposit and dissemination of scientific research documents, whether they are published or not. The documents may come from teaching and research institutions in France or abroad, or from public or private research centers.
L'archive ouverte pluridisciplinaire HAL, est destinée au dépôt et à la diffusion de documents scientifiques de niveau recherche, publiés ou non, émanant des établissements d'enseignement et de recherche français ou étrangers, des laboratoires publics ou privés. 


\title{
Robust object characterization from lensless microscopy videos
}

\author{
Olivier Flasseur $^{\dagger}$, Loïc Denis ${ }^{\dagger}$, Corinne Fournier ${ }^{\dagger}$, and Éric Thiébaut ${ }^{\ddagger}$ \\ † Univ Lyon, UJM-Saint-Etienne, CNRS, Institut d'Optique Graduate School, \\ Laboratoire Hubert Curien UMR 5516, F-42023, Saint-Etienne, France \\ $\ddagger$ Univ Lyon, Univ Lyon1, ENS de Lyon, CNRS, \\ Centre de Recherche Astrophysique de Lyon UMR5574, F-69230, Saint-Genis-Laval, France
}

\begin{abstract}
Lensless microscopy, also known as in-line digital holography, is a 3D quantitative imaging method used in various fields including microfluidics and biomedical imaging. To estimate the size and 3D location of microscopic objects in holograms, maximum likelihood methods have been shown to outperform traditional approaches based on 3D image reconstruction followed by 3D image analysis. However, the presence of objects other than the object of interest may bias maximum likelihood estimates. Using experimental videos of holograms, we show that replacing the maximum likelihood with a robust estimation procedure reduces this bias. We propose a criterion based on the intersection of confidence intervals in order to automatically set the level that distinguishes between inliers and outliers. We show that this criterion achieves a bias / variance trade-off. We also show that joint analysis of a sequence of holograms using the robust procedure is shown to further improve estimation accuracy.
\end{abstract}

\section{INTRODUCTION}

Thanks to the massive development of low-cost imaging sensors and the simultaneous increase in computational capabilities, lensless microscopy is emerging as a method of choice for time-resolved analysis of microscopic objects. It is already used in several fields where the accurate estimation of 3D location and size over time is crucial, such as in the study of fluid flows or biomedical imaging [1].

Analysis of digital holograms is traditionally performed by first reconstructing the 3D volume by light back-propagation, then analyzing this 3D volume to segment, locate and size objects [2]. However, this approach suffers from artifacts due to the imperfect reconstruction step, in particular to spatial distortions close to the image borders and spurious diffraction patterns known as twin images in holography [2]. Location and size estimates can be significantly improved by using a maximum likelihood estimator, i.e., by fitting a model of the diffraction pattern generated by the object of interest directly on the hologram rather than by reconstructing the image volume. This method is very successful for the study of isolated objects [3], [4], but some bias appears when unwanted objects in the field of view alter the hologram. These objects may have complex or unknown shapes and their influence on the hologram may be difficult to explicitly model and account for.

We suggest replacing the maximum likelihood estimator with a robust estimator so that any notable discrepancies between the model of the pattern of interest and the data due to unwanted objects have less influence on the final estimate. Section II provides a brief presentation of the lensless microscopy principle and the model of the diffraction pattern generated by spherical objects behaving as opaque objects such as droplets, cells or cocci bacteria. Section III then describes the proposed robust estimation procedure to characterize microscopic objects. Special attention is paid on the setting of the level to distinguish between inliers and outliers. An automatic criterion based on the intersection of confidence intervals is proposed to achieve a bias / variance tradeoff. Section IV illustrates the performance of the proposed method on holographic videos.

\section{CharaCterization OF MICROSCOPIC OBJECTS WITH LENSLESS IMAGING}

Lensless microscopes are based on the in-line holographic setup originally proposed by Dennis Gabor [5]. The objects are illuminated by an incident monochromatic plane wave of wavelength $\lambda$. After free-space propagation over distance $z_{p}$, a hologram $d$ is recorded by a monochromatic image sensor, see Fig. 1.

Based on diffraction theory, it is possible to model the diffraction pattern created on the hologram plane by an object of interest of given 3D shape, absorption and optical index. The case of opaque spherical objects is of particular practical interest since it covers opaque particles introduced within the flow in fluid mechanics, and colored spherical objects such as cocci bacteria or cells in flow cytometry in biology. When the distance $z_{p}$ is such that $z_{p} \gg \frac{4 \pi \cdot r_{p}^{2}}{\lambda}$, the diffraction pattern of an opaque sphere located at $\left(x_{p}, y_{p}, z_{p}\right)$, with radius $r_{p}$, is [6]:

$$
m_{p}(x, y)=\frac{\pi r_{p}^{2}}{\lambda z_{p}} \mathbf{J}_{1 \mathrm{c}}\left(\frac{2 \pi r_{p} \rho_{p}}{\lambda z_{p}}\right) \sin \left(\frac{\pi \rho_{p}}{\lambda z_{p}}\right),
$$

where $\rho_{p}=\sqrt{\left(x-x_{p}\right)^{2}+\left(y-y_{p}\right)^{2}}$ is the transversal distance to the center of the sphere and $\mathrm{J}_{1 \mathrm{c}}(u)=\mathrm{J}_{1}(u) / u$ is the cardinal Bessel function of first order. More generally, in the following $m_{\boldsymbol{\theta}}(a, b)$ denotes the intensity of the diffraction pattern generated at the pixel location $(a, b)$ on the sensor by an object of interest. This object of interest is fully characterized by a vector of parameters $\boldsymbol{\theta}$ (e.g., for an opaque sphere $\left.\boldsymbol{\theta}=\left\{x_{p}, y_{p}, z_{p}, r_{p},\right\}\right)$. 


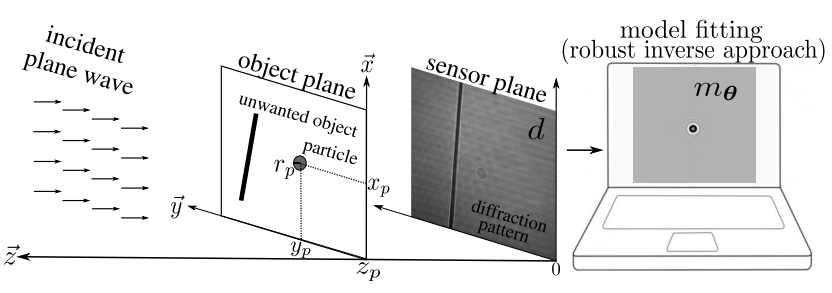

Fig. 1. Lensless microscopy setup and object fitting.

Maximum likelihood estimation of the parameters $\boldsymbol{\theta}$ of the object of interest, under the hypothesis of additive white Gaussian noise, amounts to solving a nonlinear least-squares problem [3]:

$$
\hat{\boldsymbol{\theta}}^{(\mathrm{ML})}=\underset{\boldsymbol{\theta}}{\arg \min } \sum_{a=1}^{A} \sum_{b=1}^{B} w(a, b) \cdot\left[m_{\boldsymbol{\theta}}(a, b)-d(a, b)\right]^{2},
$$

where $d(a, b)$ represents the recorded data, $w(a, b) \propto$ $1 / \epsilon(a, b)^{2}$ is a weight that is set to zero for defective pixels and that can account for a non-stationary noise variance $\epsilon(a, b)^{2}$, and $A$ and $B$ are the height and width of the sensor, in pixels.

\section{Proposed Robust estimation SCHEME}

\section{A. Robust estimation by reweighted least squares}

To reduce the impact of outliers on the estimation, Huber introduced the so-called M-estimators [7] that replace the least squares by another objective function $\rho$ in order to reduce the penalization of the largest deviations between the model and the data. Minimization problem (2) is then replaced by:

$$
\hat{\boldsymbol{\theta}}^{(\mathrm{M})}=\underset{\boldsymbol{\theta}}{\arg \min } \sum_{a=1}^{A} \sum_{b=1}^{B} w(a, b) \cdot \rho\left(\frac{m_{\boldsymbol{\theta}}(a, b)-d(a, b)}{s}\right),
$$

where $\rho$ is a non-negative, continuous and symmetric function with a minimum equal to 0 at 0 , and $s$ is a parameter that scales the residuals by setting the level that distinguishes between inliers and outliers.

Depending on the choice of the function $\rho$, the minimization problem (3) can be difficult to solve. A simple algorithmic strategy leading to a local minimizer consists in solving a sequence of least-squares problems of the form (2): the Iteratively Reweighted Least Squares (IRLS) [8]. In this scheme, the estimate $\hat{\boldsymbol{\theta}}_{k+1}$ at iteration $k+1$ is obtained by reweighting the squares with weights $w_{k}(a, b)$ derived from the residuals of iteration $k$ :

$$
\hat{\boldsymbol{\theta}}_{k+1}:=\underset{\boldsymbol{\theta}}{\arg \min } \sum_{a=1}^{A} \sum_{b=1}^{B} w_{k}(a, b) \cdot\left[m_{\boldsymbol{\theta}}(a, b)-d(a, b)\right]^{2},
$$

where the weights $w_{k}(a, b)$ are computed from the residuals $r_{k}(a, b)=m_{\boldsymbol{\theta}_{k}}(a, b)-d(a, b)$ at iteration $k$ according to an update rule that depends on the objective function $\rho$ :

$$
w_{k}(a, b)=\left.w(a, b) \cdot \frac{s}{r_{k}(a, b)} \cdot \frac{\partial \rho(u)}{\partial u}\right|_{u=r_{k}(a, b) / s} .
$$

Many different objective functions $\rho$ and their associated weight update rules have been studied and classified in the literature [9]. So-called hard-redescenders are functions $\rho$ that are constant above a given threshold, thereby leading to assign zero weights to data points that display a misfit larger than the chosen threshold. So-called soft-redescenders are functions $\rho$ whose limit at infinity is finite, thus asymptotically producing zero weights for large residual values. For lensless applications, we selected a soft-redescender to achieve a good compromise between bias reduction and loss of efficiency of the estimator. In the following, we use the Cauchy objective function:

$$
\rho(u)=\frac{1}{2} \log \left(1+u^{2}\right),
$$

which gives the following weight update:

$$
w_{k}(a, b)=w(a, b) \cdot\left[1+\left(r_{k}(a, b) / s\right)^{2}\right]^{-1} .
$$

B. Automatic tuning of parameter s: distinguishing inliers and outliers

Adequate tuning of the parameter $s$ is essential in robust estimation schemes [10]. On the one hand, if $s$ is too low, most residuals $r_{k}(a, b)$ will be (much) larger than $s$ and the weights of the corresponding data points will decrease in the following iteration, thereby discarding most of the signal of interest. In this case, the result will be an increase in the variance of the estimation. On the other hand, if $s$ is too large (much larger than the typical discrepancy of outliers), the M-estimator tends to the least squares estimator and loses its robustness properties. In this case, reduction in the bias caused by outliers is no longer obtained.

A common method to estimate the scale parameter $s$ in the case of i.i.d. noise is based on the median absolute deviation (MAD) estimator, which provides a robust estimate of the residuals standard-deviation [7]:

$$
\hat{s}^{(\mathrm{MAD})}=1.48 \cdot \operatorname{median}[|\boldsymbol{r}-\operatorname{median}(\boldsymbol{r})|],
$$

where $\boldsymbol{r}$ denotes the collection of all residuals values $r(a, b)$ for $a \in[1, A]$ and $b \in[1, B]$.

MM-estimators have been proposed to achieve high robustness to the presence of outliers while conserving high efficiency in the absence of outliers[11]. These estimators consist in two steps: (i) estimation of the scale of the residuals $s$ using an M-estimator defined by a hard-redescender $\rho$; and (ii) actual estimation of the parameters of interest $\boldsymbol{\theta}$ with a more efficient penalty function $\rho$ (such as a soft-redescender).

One drawback of these methods of setting parameter $s$ is that they do not account for the actual impact of the outliers on the estimation. When considering unwanted objects within the field of view, the location of the objects with respect to the object of interest may or may not lead to biases. In other words, while $\hat{s}^{(\mathrm{MAD})}$ is suitable in cases where the outliers are uniformly distributed in the measured signal, a more appropriate choice of $s$ could be made in cases of nonuniform distribution of outliers.

We propose to estimate parameter $s$ using a method that accounts for the actual impact of the outliers on the estimation. Starting from the value $\hat{s}^{(\mathrm{MAD})}$, which may be overconservative, we apply the intersection of confidence intervals (ICI) rule to decide up to what point parameter $s$ can be 


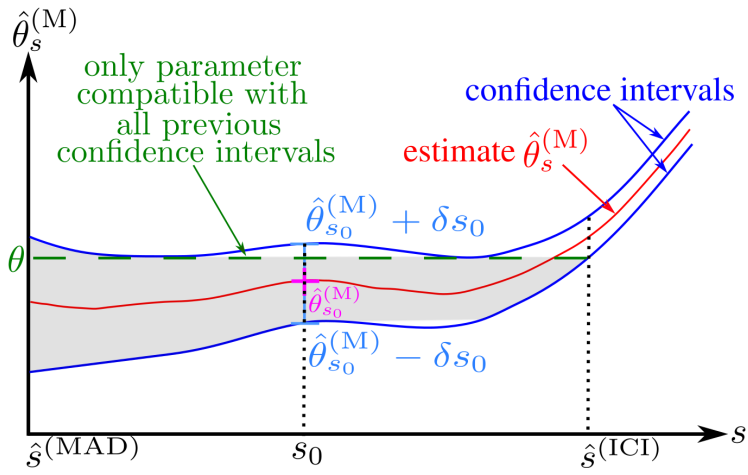

Fig. 2. Estimation of $s$ with the ICI rule.

increased to reduce estimation variance before being subject to bias due to outliers. The ICI rule has been successfully applied in image denoising to locally select the largest neighborhoods on which to perform denoising by local polynomial approximation [12]. Its application only requires knowledge of the estimate along with its standard deviation.

The vector of parameters $\boldsymbol{\theta}$ is estimated using the robust IRLS estimation scheme (4) by progressively increasing parameter $s$ until the new estimate $\hat{\boldsymbol{\theta}}_{s}^{(\mathrm{M})}$ obtained with parameter $s$ leads to a confidence interval $\left[\hat{\boldsymbol{\theta}}_{s}^{(\mathrm{M})}-\boldsymbol{\delta}_{s}, \hat{\boldsymbol{\theta}}_{s}^{(\mathrm{M})}+\boldsymbol{\delta}_{s}\right]$ with an empty intersection with the preceding confidence intervals:

$$
\begin{aligned}
& \hat{s}^{(\mathrm{ICI})}=\max \left\{s \in\left[\hat{s}^{(\mathrm{MAD})}, \infty\right) \mid\right. \\
& \left.\quad \exists \boldsymbol{\theta}, \forall s_{0} \in\left[\hat{s}^{(\mathrm{MAD})}, s\right], \boldsymbol{\theta} \in\left[\hat{\boldsymbol{\theta}}_{s_{0}}^{(\mathrm{M})}-\boldsymbol{\delta}_{s_{0}}, \hat{\boldsymbol{\theta}}_{s_{0}}^{(\mathrm{M})}+\boldsymbol{\delta}_{s_{0}}\right]\right\} .
\end{aligned}
$$

This principle is illustrated in Fig. 2 considering a single parameter $\theta$.

To build the confidence intervals, we used the Cramér-Rao Lower Bounds (CRLBs) [13]. The CRLB $\delta(i)$ represents the minimum standard-deviation for any unbiased estimator of the parameter $\theta(i)$. Under additive white Gaussian noise, the least squares estimator (2) asymptotically reaches the CRLB given by:

$$
\delta^{2}(i)=\left[I(\boldsymbol{\theta})^{-1}\right]_{i, i},
$$

where Fisher information matrix $I$ is defined by:

$$
[I(\boldsymbol{\theta})]_{i, j}=\sum_{a=1}^{A} \sum_{b=1}^{B} w(a, b) \frac{\partial m_{\boldsymbol{\theta}}(a, b)}{\partial \theta(i)} \frac{\partial m_{\boldsymbol{\theta}}(a, b)}{\partial \theta(j)}
$$

In the presence of outliers, we approximate the confidence intervals by computing the CRLB with the weights obtained by the IRLS procedure.

To reduce the computational cost of estimating parameter $s$ according to the ICI rule (9), we consider a non-uniform discretization of the set $\left[\hat{s}^{(\mathrm{MAD})}, \infty\right)$ such that two successive $s$ values lead to a given decrease in the CRLBs. In practice, each $s$ value is obtained by bisection to achieve the prescribed CRLBs reduction.

We now illustrate the tuning of parameter $s$ based on the ICI rule (9) on a simple $1 \mathrm{D}$ problem: estimating the $x_{p}$

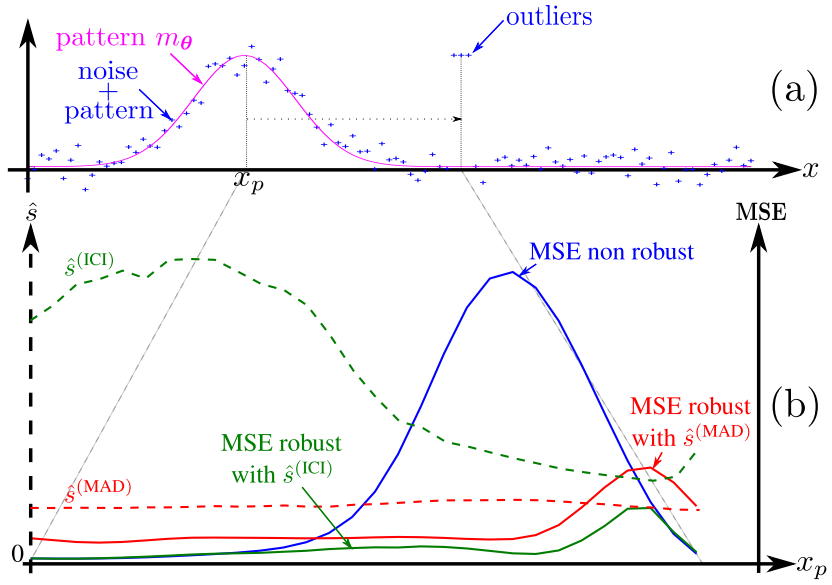

Fig. 3. Estimation of the position of a pattern by a robust approach using the MAD and the proposed ICI rule. Evolution of $\hat{s}$ and MSE are respectively in dash and solid lines.

location of a Gaussian-shaped pattern $m$. We simulate 100 noisy realizations $d$ by adding to the model a Gaussian white noise (with a peak signal-to-noise ratio of 10) and a constant outlier formed by a rectangular function with 3 samples in width (with a peak signal-to-noise ratio of 1), see Fig. 3(a).

The ICI rule leads to values of $s$ that are about 5 times larger than $\hat{s}^{(\mathrm{MAD})}$ when the outlier is too far to introduce a significant bias, and to values of $s$ close to that of $\hat{s}^{\text {(MAD) }}$ when the outlier affects the estimation, see Fig. 3(b). When $\hat{s}^{(\mathrm{ICI})}$ is higher than $\hat{s}^{(\mathrm{MAD})}$ (resulting in a weaker penalization of the residuals), the mean square error (MSE) on the location of the pattern is reduced by a factor between 2 and 4 . In practice, the empirical standard-deviation reaches the CLRB when the outlier has little impact on the estimate. A difference of a few tens of percent is observed when the constant outlier has an impact on the estimate since the estimator is no longer asymptotically unbiased. Determination of $s$ with an ICI rule is still possible. In conclusion, choosing the parameter $s$ with the ICI rule improves the mean square error compared to a MAD estimator.

\section{ApPliCATION TO LENSLESS MiCROSCOPY VIDEOS}

In this section, the performance of the robust estimate scheme applied in lensless microscopy is evaluated on two lensless imaging applications. The goal is to accurately estimate the radius $r_{p}$ of spherical objects. We also evaluate the performance of a robust estimation performed jointly over several consecutive frames (super-resolution). In these examples, the image formation model $m_{\theta}$ is non-linear with respect to the parameters $\boldsymbol{\theta}$, as given by equation (1). As a result, the minimization problem (2) and each IRLS iteration in (4) is solved using a few iterations of Levenberg-Marquardt algorithm. The original videos and the results of the robust estimation are available online at http://perso.univ-st-etienne. fr/deniloic/robust_lensless_video.html.

\section{A. Holographic video of an object of constant size}

We consider a 200 frames video of a slightly moving particle located near an unwanted object. The object of interest 


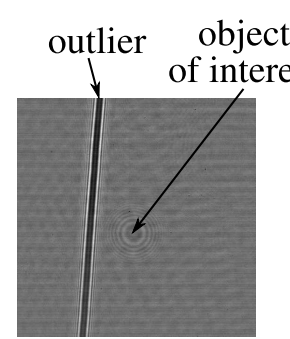

hologram

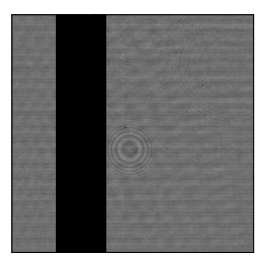

manual mask

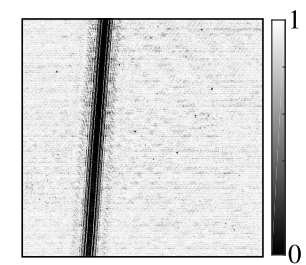

weights from IRLS
Fig. 4. Calibrated particle near an outlier - example of a frame.

is a circular chromium deposit on a glass plate, with a calibrated radius of $50 \mu \mathrm{m}$ (Optimask ${ }^{\mathrm{TM}}$, diameter $\pm 1 \mu \mathrm{m}$, roundness error $\pm 0.25 \mu \mathrm{m})$. The unwanted object is a fixed human hair [14].

Since the outlier is fixed throughout the video, it is possible to roughly remove outlier pixels by applying a static binary weighting mask $w(a, b)=0$ if pixel $(a, b)$ is in the masked region, $w(a, b)=1$ otherwise. Figure 4 shows two frames from the video and the manual removal of the outlier by masking. Due to the mask $w$, part of the signal due to the particle of interest (diffraction rings) is not accounted for.

Since the radius $r_{p}$ of the particle remains constant during acquisition, it is possible to jointly estimate this parameter in several consecutive frames. This super-resolution approach has already been successfully applied in lensless microscopy considering the non-robust minimization problem (2) [14]. We propose to combine this super-resolution approach with a robust estimation scheme (minimization problem (4)) considering 40 stacks of 5 successive frames.

In each frame the vector of parameters $\boldsymbol{\theta}$ is estimated by least squares fitting, Eq. (2), with constant weights (hereafter denoted single - without mask) or with the binary mask (denoted single - with mask), and using the robust $\mathrm{M}$ estimator, Eq. (4) and $\hat{s}^{\text {(MAD) }}$ (denoted single - robust). In the case of a joint estimation, the term single is replaced by the term joint in the denomination of the methods tested.

Figure 4 is an example of the weights used for the last IRLS iteration. The weights are close to a segmentation of the pixels impacted by the unwanted object. The diffraction rings characterizing the particle of interest are well preserved (weights close to 1). Figure 5 shows the evolution of the estimated radius during the IRLS iterations for the first 5 frames of the video. This shows that the algorithm converges in a few iterations and that the joint estimate of the radius carried out by super-resolution is very close to the ground truth equal to $50 \mu \mathrm{m}$.

Table I lists the mean estimated radius $\left\langle\hat{r}_{p}^{(\mathrm{M})}\right\rangle$, the corresponding standard-deviation $\sigma$ and the CRLB $\delta$ obtained with $\hat{s}^{(\mathrm{MAD})}$ for each method tested. The manual removal of the outliers appears to reduce the estimation bias. The bias is further reduced when a robust weighting of residuals by IRLS is applied since it preferentially eliminates the outliers while retaining the signal of interest. As a result, the estimation using a robust approach leads to a very small increase in CLRB

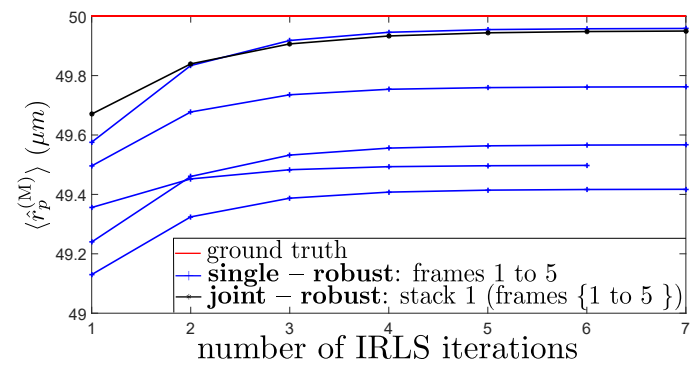

Fig. 5. Estimated radius as a function of the number of IRLS iterations.

TABLE I

MEAN ESTIMATED RADIUS, STANDARD-DEVIATION $\sigma$ AND CRLB $\delta$ FOR THE DIFFERENT METHODS TESTED

\begin{tabular}{cccc}
\hline Method: & $\left\langle\hat{r}_{p}^{(\mathrm{M})}\right\rangle(\mu m)$ & $\sigma(\mu m)$ & $\delta(\mu m)$ \\
\hline single - without mask & 49.71 & 0.31 & 0.014 \\
single - with mask & 49.78 & 0.25 & 0.016 \\
single - robust & 49.91 & 0.26 & 0.015 \\
\hline joint - without mask & 49.71 & 0.13 & $0.014 / \sqrt{5}$ \\
joint - with mask & 49.78 & 0.08 & $0.016 / \sqrt{5}$ \\
joint - robust & 49.93 & 0.09 & $0.015 / \sqrt{5}$ \\
\hline
\end{tabular}

which is negligible compared with the reduction of the bias.

The combination of a robust estimation method with a super-resolution scheme (joint - robust) exploiting the information redundancy between different frames significantly reduce the estimation standard-deviation. In agreement with statistical results [15], we observed a decrease in the standarddeviation by a factor at least equal to $\sqrt{5}$. This joint robust estimation approach enables accurate (i.e. low bias and low standard-deviation) estimates of the radius of the holographic objects and thus improves the overall performance of a conventional least squares estimation process.

\section{B. Holographic video of evaporating droplets}

We used a 160 frames video of an evaporating diethyl ether droplet dispersed in a homogeneous and nearly isotropic turbulence that is tracked over time. As the particle evaporates, it produces a plume of vapor that diffuses around it. The vapor plume creates outliers since its signal mixes with the signal produced by the particle of interest. Other evaporating particles passing close to the particle of interest also act as outliers. The evaporation rate of these particles was recently studied using the least squares approach (2) [16].

Figure 6(b) shows two frames from the video and the 2D trajectory of the particle over time (superimposed color line).

Figure 6(a) shows the evolution of the rescale factor of the residuals obtained with a MAD estimator and with the proposed ICI rule as well as the IRLS weights for two different frames. The ICI rule leads to a larger value of $s$ than MAD but without notably modifying the estimation of the radius $r_{p}$. This is the case when outliers do not disturb the estimate like in frame 60 (left column) since the particle of interest is isolated and the plume of vapor is rather small. In contrast, $\hat{s}^{(\mathrm{MAD})}$ and $\hat{s}^{\text {(ICI) }}$ are very close when the particle of interest is strongly disturbed by outliers. This is illustrated by frame 97 (right 
(a)

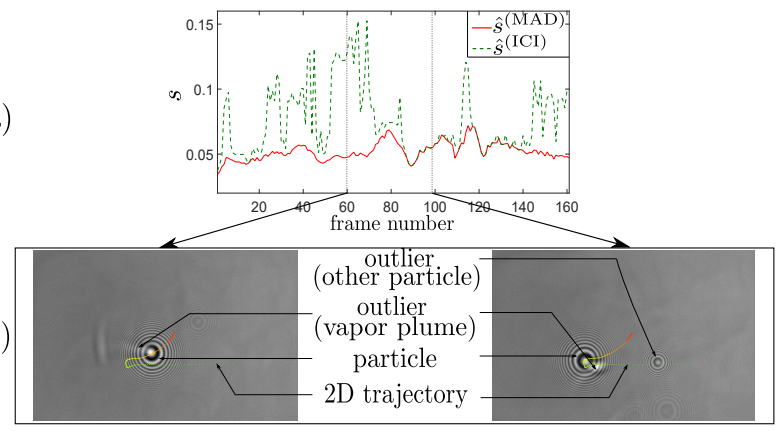

(c)
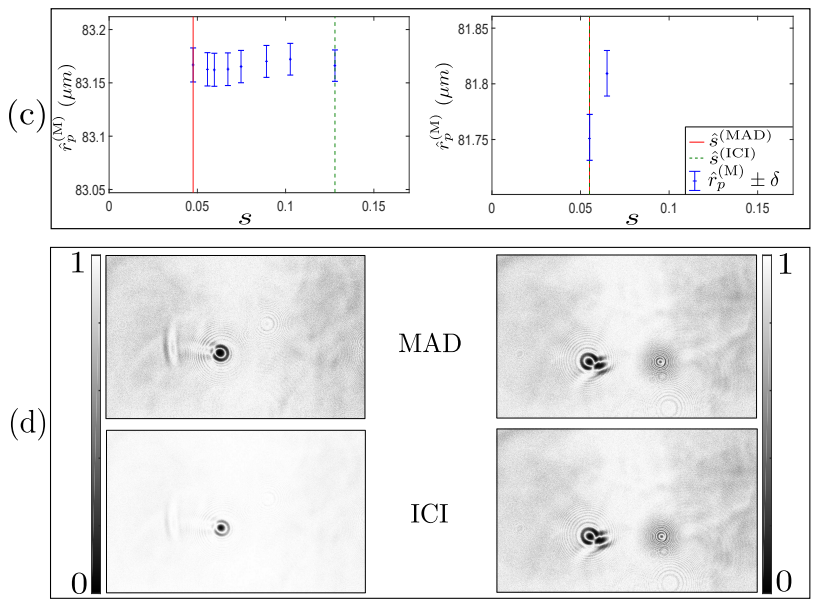

Fig. 6. Robust detection and tracking of an evaporating droplet. (a) evolution of $\hat{s}^{\text {(MAD) }}$ and $\hat{s}^{\text {(ICI) }}$ (b), (c) and (d) respectively give the holograms, confidence interval diagrams, and robust weighting maps for two video frames. (holographic video credit: Fluid Mechanics and Acoustics Laboratory LMFA, CNRS-Université de Lyon, France)

column) where another evaporating particle is close to the particle of interest and disturbs its signal. These observations underline the fact that the MAD estimator only accounts for the presence of outliers strongly deviating from the model but does not account for their spatial localization and hence, their actual impact on the estimate. Figure 6(c) illustrates the evolution of confidence intervals for increasing values of $s$. Figure 6(d) shows the weights obtained at the end of the IRLS procedure for $\hat{s}^{(\mathrm{MAD})}$ (first row) and $\hat{s}^{\text {(ICI) }}$ (second row). On frame $60, \hat{s}^{(\mathrm{ICI})}$ is significantly larger than $\hat{s}^{(\mathrm{MAD})}$, leading to larger weights, while on frame $97 \hat{s}^{(\mathrm{MAD})}=\hat{s}^{(\mathrm{ICI})}$ and the weights are identical.

Figure 7 shows the evolution of the estimated radius over time with the three methods of estimation tested. The robust estimation scheme leads to an average increase of $0.4 \mu \mathrm{m}$ in the estimated radius. We also observed that it reduces the estimation standard-deviation. These experimental results are explained by the fact that the moving outliers (vapor plume plus other particle) cause a non-stationary disturbance of the signal of interest over time. The robust approach considering a MAD or ICI rule to set the parameter $s$ lead to close results in this application. This is due to the fact that the weighting matrix obtained with $\hat{s}^{(\mathrm{MAD})}$ already guarantees a high level of efficiency (around $90 \%$ of the maximum efficiency obtained without reweighting).

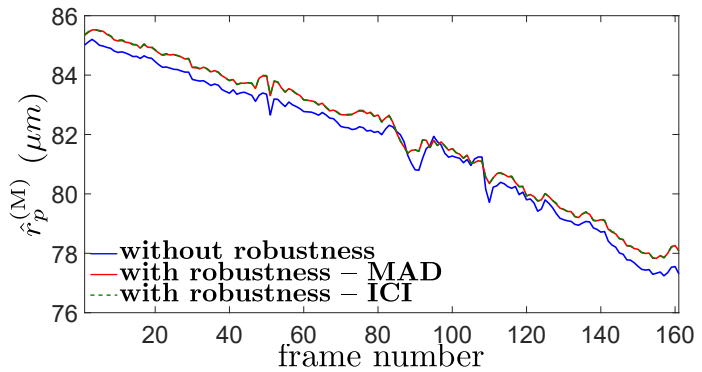

Fig. 7. Estimated radius over time with the different methods tested.

\section{CONClusion}

We have shown that the use of a robust approach to estimate the 3D position and size of objects in lensless microscopy videos reduces the estimation bias caused by unwanted objects. We proposed a simple method to estimate the scale parameter that distinguishes between inliers and outliers in the robust estimation scheme. This method is based on an intervals confidence intersection rule and achieves a better bias / variance trade-off than the usual MAD rule. Finally, we have shown that the combination of the proposed robust approach with a joint estimation scheme also reduces the standard-deviation of the estimation and consequently improves the overall performance of the method.

Work supported by CNRS grant DETECTION (DEFI IMAG'In 2015)

\section{REFERENCES}

[1] A. Greenbaum et al., "Imaging without lenses: achievements and remaining challenges of wide-field on-chip microscopy," Nature methods, vol. 9, no. 9, 2012.

[2] T. Kreis, Handbook of holographic interferometry: optical and digital methods. John Wiley \& Sons, 2006.

[3] F. Soulez et al., "Inverse-problem approach for particle digital holography: accurate location based on local optimization," JOSA A, vol. 24, no. 4, 2007

[4] C. Wang et al., "Holographic characterization of protein aggregates," $J$. of pharmaceutical sciences, vol. 105, no. 3, 2016.

[5] D. Gabor, "A new microscope principle," Nature, no. 1161, 1948.

[6] G. A. Tyler and B. J. Thompson, "Fraunhofer holography applied to particle size analysis a reassessment," J. of Modern Optics, vol. 23, no. 9, 1976.

[7] P. J. Huber, Robust statistics. Springer, 2011.

[8] P. W. Holland and R. E. Welsch, "Robust regression using iteratively reweighted least-squares," Communications in Statistics-theory and Methods, vol. 6, no. 9, 1977.

[9] J. E. Dennis Jr and R. E. Welsch, "Techniques for nonlinear least squares and robust regression," Communications in Statistics-Simulation and Computation, vol. 7, no. 4, 1978.

[10] P. J. Rousseeuw and A. M. Leroy, Robust regression and outlier detection. John Wiley \& sons, 2005, vol. 589.

[11] V. J. Yohai, "High breakdown-point and high efficiency robust estimates for regression," The Annals of Statistics, 1987.

[12] V. Katkovnik, K. Egiazarian, and J. Astola, "Adaptive window size image de-noising based on intersection of confidence intervals (ici) rule," J. of Mathematical Imaging and Vision, vol. 16, no. 3, 2002.

[13] A. Stuart and J. Odd, Kendall's Advanced Theory of Statistics : Distribution Theory. London, Arnold, 1994.

[14] N. Verrier and C. Fournier, "Digital holography super-resolution for accurate three-dimensional reconstruction of particle holograms," Optics letters, vol. 40, no. 2, 2015.

[15] D. Robinson and P. Milanfar, "Statistical performance analysis of superresolution," IEEE Trans. on Image Processing, vol. 15, no. 6, 2006.

[16] J. L. Marié et al., "Digital holographic measurement of the lagrangian evaporation rate of droplets dispersing in a homogeneous isotropic turbulence," Experiments in Fluids, vol. 58, no. 2, 2017. 